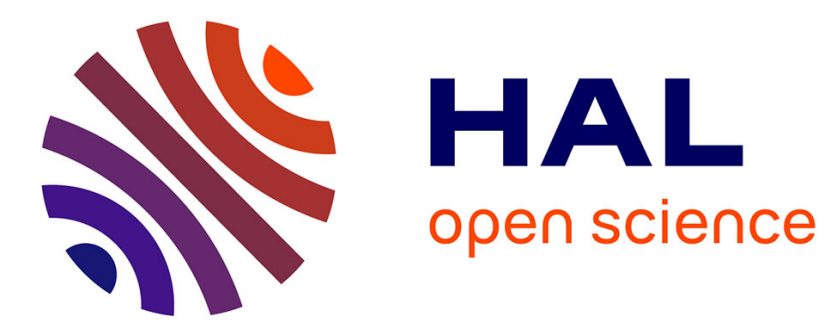

\title{
A note on: An optimal batch size for an imperfect production system with quality assurance and rework
}

\author{
Udo Buscher, Steffen Rudert, Christian Schwarz
}

\section{To cite this version:}

Udo Buscher, Steffen Rudert, Christian Schwarz. A note on: An optimal batch size for an imperfect production system with quality assurance and rework. International Journal of Production Research, 2009, 47 (24), pp.7063-7067. 10.1080/00207540802317723 . hal-00530218

\section{HAL Id: hal-00530218 \\ https://hal.science/hal-00530218}

Submitted on 28 Oct 2010

HAL is a multi-disciplinary open access archive for the deposit and dissemination of scientific research documents, whether they are published or not. The documents may come from teaching and research institutions in France or abroad, or from public or private research centers.
L'archive ouverte pluridisciplinaire HAL, est destinée au dépôt et à la diffusion de documents scientifiques de niveau recherche, publiés ou non, émanant des établissements d'enseignement et de recherche français ou étrangers, des laboratoires publics ou privés. 


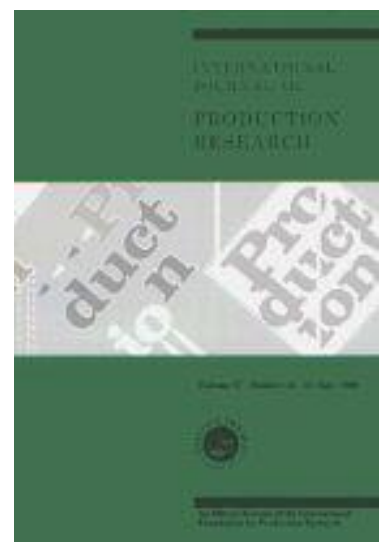

A note on: An optimal batch size for an imperfect production system with quality assurance and rework

\begin{tabular}{|r|l|}
\hline Journal: & International Journal of Production Research \\
\hline Manuscript ID: & TPRS-2008-IJPR-0173.R1 \\
\hline Manuscript Type: & Technical Note \\
\hline Author: & 19-Jun-2008 \\
\hline & $\begin{array}{l}\text { Complete List of Authors: } \\
\text { Rudert, Steffen; Dresden University of Technology, Faculty of } \\
\text { Business Management Management and Economics, Department of Industrial } \\
\text { Management } \\
\text { Schwarz, Christian; Dresden University of Technology, Faculty of } \\
\text { Business Management and Economics, Department of Industrial } \\
\text { Management }\end{array}$ \\
\hline Keywords: & LOT SIZING, RE-MANUFACTURING, QUALITY ASSURANCE \\
\hline Keywords (user): & LOT SIZING, RE-MANUFACTURING \\
\hline & \\
\hline
\end{tabular}

\section{\$) ScholarONE" \\ Manuscript Central}




\section{RESEARCH NOTE}

\section{A note on: An optimal batch size for an imperfect production system with quality assurance and rework}

Udo Buscher $^{\mathrm{a}^{*}}$, Steffen Rudert ${ }^{\mathrm{a}}$ and Christian Schwarz ${ }^{\mathrm{a}}$

${ }^{a}$ Faculty of Business Management and Economics, Department of Industrial Management, Dresden University of Technology, 01062 Dresden, Germany

In Ojha et al. 2007 three models are introduced in the context of imperfect production systems. By analysing the proposed models we identify some problems. In particular we focus on six issues of the models developed in Ojha et al. and propose some modifications. All corrections are presented in detail and the numerical example of Ojha et al. is used to show the influence of the issues addressed in this paper.

Keywords: Imperfect production, Lot sizing, Remanufacturing

*Corresponding author. Email: udo.buscher@tu-dresden.de

\section{Analysis}

In Ojha et al., a static deterministic model with imperfect production is considered. Raw materials are received from a supplier, processed and delivered to the customer. Three scenarios are distinguished. Within the most complex one, multiple lots using a batch of raw material and each production lot is delivered to the customer in multiple instalments. Furthermore, two special cases of this model are considered. In the second and third model all produced items of a lot are delivered in a single instalment. The third model additionally applies a lot-for-lot policy, i.e. the raw material is ordered in quantity $Q_{r}$ that is required for one production run. In spite of the contribution of Ojha et al. understanding imperfect production in the context of inventoryproduction-delivery systems, some issues addressed in their paper remain unclear. In the following we discuss six issues in more detail. 
Firstly, Ojha et al. assume merely that production rate $P$ must be higher than demand rate $D_{f}$. However, when dealing with imperfect production, this does not necessarily avoid shortages in the system. Using the same equipment, rework is done directly after production. Since sequential movement is assumed additionally, the sum of production and rework time $\left(t_{1}+t_{2}\right)$ must not exceed the cycle time $T=Q_{f} / D_{f}$. Recall that production uptime $t_{1}$ is defined as $Q_{f} / P$ and the rework time $t_{2}$ is given by $\left(\beta Q_{f}\right) / P$, the aforementioned condition only holds if $P \geq(1+\beta) D_{f}$ is valid. Since this is a general condition, it affects all three models developed by Ojha et al.

Secondly, the price for an item of raw material in Ojha et al. is $C$. In addition, the conversion factor $f_{0}$ specifies how many finished goods can be made out of one raw material item. It should be noted that $C$ is used as raw material price for a raw material part as well as for the raw material price of a finished product. This is only true for the special case of $f_{0}=1$. Therefore, we have to differentiate between $C_{r}$, the price for one unit raw material, and $C_{f}$, the effective value of raw material for a finished product. The relation of $C_{r}$ and $C_{f}$ is obviously $C_{f}=C_{r} / f_{0}$. In this context, the inventory carrying cost for reworkable items $I_{R E}$ and for finished products $I_{F}$ are affected.

Thirdly, we address the finished goods inventory carrying cost where a production lot is distributed to the customer in multiple instalments. In this case the finished goods inventory carrying cost consists of those costs which are incurred during production and those which are incurred during delivery: $I_{F}=I_{F, \text { prod }}+I_{F, \text { del }}$. While $I_{F, \text { prod }}$ is calculated correctly, we examine $I_{F, d e l}$. Following Ojha et al. the values of non-defective items and reworked items are $C_{f}+V$ and $C_{f}+2 V$, respectively. Unfortunately, this fact is ignored while calculating $I_{F, d e l}$. The average cost per unit of the finished product is

$$
\frac{\left(C_{f}+V\right)(1-\beta) Q_{f}+\left(C_{f}+2 V\right) \beta Q_{f}}{Q_{f}}=C_{f}+V(1+\beta) .
$$

Thus, the correct finished goods inventory carrying costs are

$$
I_{F}=i\left(C_{f}+V\right) \frac{D_{f} Q_{f}}{2 P}[1+\beta(1-\beta)]+i\left[C_{f}+V(1+\beta)\right] \frac{x}{2}(m-1) \text {; }
$$

or

$$
I_{F}=i\left(C_{f}+V\right)\left(\frac{D_{f} Q_{f}}{2 P}[1+\beta(1-\beta)]+\frac{x}{2}(m-1)\right)+\frac{\beta i x V}{2}(m-1) .
$$

Fourthly, usually in static deterministic inventory-models the cost function is formulated in $\$$ per year. However, Ojha et al. specify inspection, late delivery and lost production costs as cycle cost. The corresponding expressions have to be divided by the cycle length $T$. The correct expressions are $C_{I}=C_{i} D_{f} ; C_{L}=\left(\beta C_{l} D_{f}\right) / P ; C_{L P}=\beta D_{f} V$. In consequence, these cost components have no impact on the optimal values of the decision variables. 
In the following the issues described above are incorporated into the total cost functions. As a consequence the functions of $Q^{*}, n^{*}$ and $m^{*}$ need to be adjusted slightly.

$$
\begin{aligned}
& T C^{I}(m, n)=\frac{i m x C_{f}}{2}\left[\frac{D_{f}}{P}+n-1\right]+\frac{D_{f}}{m x}\left(\frac{A_{r}}{n}+A_{f}\right) \\
& +i\left(C_{f}+V\right) \frac{m x D_{f}}{2 P}[1+\beta(1-\beta)]+i\left(C_{f}+V(1+\beta)\right) \frac{x}{2}(m-1) \\
& +V D_{f}(1+\beta)+\frac{i m x \beta D_{f}}{2 P}(1+\beta)\left(C_{f}+V\right)+\frac{i m x \beta^{2} D_{f} V}{2 P} \\
& +C_{i} D_{f}+\frac{\beta C_{l} D_{f}}{P}+\beta D_{f} V \\
& m^{I^{*}}=\sqrt{\frac{A_{f} D_{f}}{\Delta_{1} x}} \\
& n^{I^{*}}=\sqrt{\frac{2 \Delta_{1} A_{r}}{i x A_{f} C_{f}}} \\
& \Delta_{1}=\frac{i x C_{f}}{2}\left(\frac{D_{f}}{P}-1\right)+\frac{i x D_{f}}{2 P}\left(C_{f}+V\right)[1+\beta(1-\beta)] \\
& +\frac{i x}{2}\left(C_{f}+V(1+\beta)\right)+\frac{\beta i x D_{f}}{2 P}(1+\beta)\left(C_{f}+V\right)+\frac{\beta^{2} i x D_{f} V}{2 P} \\
& T C^{I I}\left(Q_{f}, n\right)=\frac{i C_{f} Q_{f}}{2}\left[\frac{D_{f}}{P}+n-1\right]+\frac{D_{f}}{Q_{f}}\left(\frac{A_{r}}{n}+A_{f}\right) \\
& +\frac{i Q_{f} D_{f}}{2 P}[1+\beta(1-\beta)]\left(C_{f}+V\right)+V D_{f}(1+\beta) \\
& +\frac{i \beta Q_{f} D_{f}}{2 P}(1+\beta)\left(C_{f}+V\right)+\frac{i \beta^{2} Q_{f} D_{f} V}{2 P} \\
& +C_{i} D_{f}+\frac{\beta C_{l} D_{f}}{P}+\beta D_{f} V \\
& Q_{f}^{I I *}(n)=\sqrt{\frac{D_{f}\left(\frac{A_{r}}{n}+A_{f}\right)}{\frac{i n C_{f}}{2}+\Delta_{2}}}
\end{aligned}
$$




$$
\begin{gathered}
Q_{f}^{I *^{*}}=\sqrt{\frac{A_{f} D_{f}}{\Delta_{2}}} \\
n^{I I^{*}}=\sqrt{\frac{2 \Delta_{2} A_{r}}{i A_{f} C_{f}}} \\
\Delta_{2}=\frac{i C_{f}}{2}\left(\frac{D_{f}}{P}-1\right)+i\left(C_{f}+V\right) \frac{D_{f}}{2 P}[1+\beta(1-\beta)]+\frac{\beta^{2} i D_{f} V}{2 P} \\
+\frac{\beta i D_{f}}{2 P}(1+\beta)\left(C_{f}+V\right) \\
T C^{I I I}\left(Q_{f}\right)=\frac{i Q_{f} D_{f} C_{f}}{2 P}+\frac{D_{f}}{Q_{f}}\left(A_{r}+A_{f}\right) \\
+\frac{i Q_{f} D_{f}}{2 P}[1+\beta(1-\beta)]\left(C_{f}+V\right)+V D_{f}(1+\beta) \\
\Delta_{3}=\frac{i C_{f} D_{f}}{P}+i\left(C_{f}+V\right) \frac{D_{f}}{P}[1+\beta(1-\beta)]+\frac{\beta i D_{f}}{P}(1+\beta)\left(C_{f}+V\right) \\
+C_{f} D_{f} D_{f}+\frac{\beta C_{l} D_{f}}{P}+\beta D_{f} V \\
Q_{f}^{I I I^{*}}=\sqrt{\frac{2 D_{f}}{\Delta_{3}}\left(A_{r}+A_{f}\right)} \\
+\beta)\left(C_{f}+V\right)+\frac{i Q_{f} V}{2 P}
\end{gathered}
$$

Fifthly, taking the first derivative of $T C^{I}(m, n)$ with respect to $m$ we see that the optimal value of $m$ depends on the given $n$, thus $m^{*}(n)$. Conversely, the optimal value of $n$ is also a function of $m$. Therefore, it is not sufficient to verify only the four combinations $T C^{I}\left(\left\lfloor m^{*}\right\rfloor\left\lfloor n^{*}\right\rfloor\right), \quad T C^{I}\left(\left\lfloor m^{*}\right\rfloor,\left\lceil n^{*}\right\rceil\right), \quad T C^{I}\left(\left[m^{*}\right\rceil,\left\lfloor n^{*}\right\rfloor\right)$, $T C^{I}\left(\left[m^{*}\right],\left[n^{*}\right]\right)$ to derive the optimal solution. For example, a Branch\&Bound-procedure could be applied to obtain the optimal integer values $m_{\text {opt }}$ and $n_{\text {opt }}$. The first step is to round $m^{I^{*}}$ and $n^{I^{*}}$ to the nearest integer value and to insert them in (3). The resulting costs $C_{b}$ represent the first upper bound. Now assume that, based on (3), merely $m$ is pre-specified and we are interested in determining the lowest possible costs for given $m$-values. Obviously, this requires us to solve the first order condition $\partial\left(T C^{I}(m, n)\right) / \partial(n)=0$ for $n$ : 


$$
n(m)=\frac{1}{m} \sqrt{\frac{2 A_{r} D_{f}}{i x^{2} C_{f}}}
$$

Substituting of this formula into (3) provides

$$
T C(m)=\Delta_{1} m+\frac{A_{f} D_{f}}{x m}+\Delta_{4}
$$

with

$$
\begin{aligned}
\Delta_{4} & =-i\left(C_{f}+V(1+\beta)\right) \frac{x}{2}+D_{f} V+\beta D_{f} V+C_{i} D_{f}+\frac{\beta C_{l} D_{f}}{P} \\
& +\beta D_{f} V+\sqrt{2 i C_{f} A_{r} D_{f}} .
\end{aligned}
$$

Since (3) is convex in $m$, we are able to establish a lower bound $m_{l}$ and an upper bound $m_{u}$ within which the optimal integer value of $m$ is located. To compute both boundary values, we equate the costs $C_{b}$ with the right-hand side of (15) and solve the resulting expression for $m$. In doing so, we obtain a quadratic equation which provides the following two integer values:

$$
m_{l}=\max \left(1 ;\left\lceil\alpha_{1}-\alpha_{2}\right\rceil\right) \text { and } m_{u}=\max \left(1 ;\left\lfloor\alpha_{1}+\alpha_{2}\right\rfloor\right)
$$

with

$$
\alpha_{1}=-\frac{\Delta_{4}-C_{b}}{2 \Delta_{1}} \text { and } \quad \alpha_{2}=\sqrt{\left(\frac{\Delta_{4}-C_{b}}{2 \Delta_{1}}\right)^{2}-\frac{A_{f} D_{f}}{\Delta_{1}}} \text {. }
$$

Due to the convexity of $T C$ in $n$, it is possible to calculate the optimal integer $n$-value for a given $\mathrm{m}$ by $\lfloor n(m)\rfloor$ or $\lceil n(m)\rceil$. For all $m$-values between the lower and upper bound the optimal $n$-value is determined by comparing the resulting costs. Each time a new temporary optimal solution is found, we update the bounds $m_{l}$ and $m_{u}$ by using cost $T C^{I}$ as the new $C_{b}$-value. This successive tightening of these bounds offers - in contrast to using static bounds - the potential to considerably reduce the solution effort needed to ascertain the cost minimizing solution. After examining all relevant pairs $(m, n)$, the best solution so far represents the optimal solution for the underlying planning problem.

Sixthly, in the second model of Ojha et al. the lot size $Q_{f}$ and the number $n$ of production lots have to be determined. Obviously, $n_{\text {opt }}$ has to be an integer value. Therefore, Ojha et al. compare $T C^{I I}\left(Q^{*},\left\lfloor n^{*}\right\rfloor\right)$ and $T C^{I I}\left(Q^{*},\left[n^{*}\right]\right)$ to find $n_{\text {opt }}$. However, it would be favorable to calculate the optimal $Q_{f}$ for a given value of $n$ and compare the resulting cost afterwards, i.e. $T C^{I I}\left(Q\left(\left\lfloor n^{*}\right\rfloor\right),\left\lfloor n^{*}\right\rfloor\right)$ and $T C^{I I}\left(Q\left(\left[n^{*}\right\rceil\right),\left\lceil n^{*}\right\rceil\right)$. 


\section{Numerical example}

For better understanding the impact of the issues described above, we use the numerical example of Ojha et al. The problem parameters are: $\beta_{1}=0.05 ; E_{1}=0.1 ; E_{2}=0.1 ; A_{f}=50$ [\$ per setup]; $A_{r}=150$ [\$ per order]; $C_{r}=2$ [\$ per unit raw material]; $C_{i}=0.2$ [\$ per unit]; $C_{l}=0.1$ [\$ per year]; $D_{f}=2000$ [units per year]; $f_{0}=0.95 ; i=0.18$ [per year]; $P=3000$ [units per year]; $V=1$ [\$ per unit]; $x=20$ [units]; $C_{f}=C_{r} / f_{0}=2.11$. The procedure to get the optimal solution for model I is shown in the following table.

**** Place table 1 here $* * * *$

The procedure proposed by Ojha et al. does not consider the combination $m=22$ and $n=3$, which is the optimal solution.

The results using the formulation presented in this paper and those of Ojha et al. are given in table 2. Whereas a plus sign is used to indicate the results obtained in this note, a hash marks the results of Ojha et al. It should be noted that $T C^{\#}$ is calculated by inserting the production policy derived in Ojha et al. into the cost functions derived in this note to get an impression of the induced cost deviations for the given example.

**** Place table 2 here $* * * *$

Due to the corrected total cost function, the optimal expressions of the decision variables $m, n$ and $Q_{f}$ show significant differences. Finally, it is important to point out that the main idea and contribution of the paper of Ojha et al. have a great value.

\section{References}

Ojha, D., Sarker, B.R., and Biswas, P., 2007. An optimal batch size for an imperfect production system with quality assurance and rework. International Journal of Production Research, 45 (14), 3191-3214. 
Table 1. Iterations of Branch\&Bound for model I.

\begin{tabular}{ccccccc}
\hline iteration & $m$ & $n$ & $T C^{I}(m, n)$ & $C_{b}$ & range of $m$ & current $m_{\text {opt }} ; n_{\text {opt }}$ \\
\hline start & 23 & 3 & 3865.97 & 3865.97 & $21 \leq m \leq 25$ & $23 ; 3$ \\
1 & 21 & 2 & 3905.37 & 3865.97 & $21 \leq m \leq 25$ & $23 ; 3$ \\
2 & 21 & 3 & 3865.90 & 3865.90 & $21 \leq m \leq 25$ & $21 ; 3$ \\
3 & 22 & 2 & 3895.26 & 3865.90 & $21 \leq m \leq 25$ & $21 ; 3$ \\
4 & 22 & 3 & 3864.99 & 3864.99 & $22 \leq m \leq 24$ & $22 ; 3$ \\
5 & 23 & 2 & 3887.51 & 3864.99 & $22 \leq m \leq 24$ & $22 ; 3$ \\
6 & 23 & 3 & 3865.97 & 3864.99 & $22 \leq m \leq 24$ & $22 ; 3$ \\
7 & 24 & 2 & 3881.81 & 3864.99 & $22 \leq m \leq 24$ & $22 ; 3$ \\
8 & 24 & 3 & 3868.59 & 3864.99 & $22 \leq m \leq 24$ & $22 ; 3$ \\
\hline
\end{tabular}

Table 2 Comparison of results.

\begin{tabular}{ccccccccc}
\hline & $Q_{f}^{+}$ & $Q_{f}^{\#}$ & $n^{+}$ & $n^{\#}$ & $m^{+}$ & $m^{\#}$ & $T C^{+}$ & $T C^{\#}$ \\
\hline I & 440 & 340 & 3 & 4 & 22 & 17 & 3864.99 & 3885.87 \\
II & 671 & 443 & 2 & 3 & - & - & 3705.29 & 3741.48 \\
III & 1045 & 757 & - & - & - & - & 3725.23 & 3765.45 \\
\hline
\end{tabular}

